IASSNS-HEP-97/62,hep-th/9706046

\title{
Entropy of Non-Extreme Rotating Black Holes in String Theories
}

\author{
Donam Youm * \\ School of Natural Sciences, Institute for Advanced Study \\ Olden Lane, Princeton, NJ 08540
}

(March 1997)

\begin{abstract}
We formulate the Rindler space description of rotating black holes in string theories. We argue that the comoving frame is the natural frame for studying thermodynamics of rotating black holes and statistical analysis of rotating black holes gets simplified in this frame. We also calculate statistical entropies of general class of rotating black holes in heterotic strings on tori by applying $D$-brane description and the correspondence principle. We find at least qualitative agreement between the Bekenstein-Hawking entropies and the statistical entropies of these black hole solutions.
\end{abstract}

04.60.-m,04.62.+v,04.70.-s,04.70.Bw,04.70.Dy,05.20.-y,05.70.-a,11.27.+d

*E-mail address: youm@sns.ias.edu 


\section{INTRODUCTION}

Past couple of years have been revolutionary period for understanding one of challenging problems in quantum theory of gravity. Namely, we are now able to reproduce BekensteinHawking entropy [1-5] of special class of black holes through stringy statistical calculations without encountering infinities associated with non-renomalization of quantum gravity or information loss problems [6].

This was first anticipated in Ref. [7], where it is predicted that since string theory is a finite theory of quantum gravity the calculation of partition function in the canonical quantum gravity of superstring theories would yield finite statistical entropy of black holes. In this description, the microscopic degenerate degrees of freedom responsible for non-zero statistical entropy of black holes are oscillating degrees of freedom of strings which are in thermal equilibrium with black hole environment [8]. (Only strings that contribute to statistical entropy are those that are somehow entangled with the event horizon and therefore look like oscillating open strings whose ends are attached to the event horizon from the point of view of external observers.)

The explicit calculation of the degeneracy of (microscopic) string states that are associated with (macroscopic) black hole became possible with identification [9] of subsets of black hole solutions in effective string theories with massive string states and the level matching condition [10], which justifies such identification. The qualitative agreement (up to a factor of numerical constant of order 1) was first observed in Ref. [11], where the Bekenstein-Hawking entropy of NS-NS electric charged, non-rotating, black holes in heterotic string which is calculated at the stretched horizon is compared with degeneracy of massive perturbative string states.

Precise calculation of statistical entropy was made possible with construction of general class of black hole solutions (for example those constructed in Refs. [12,13]) which have nonzero event horizon area in the BPS limit. Such solutions necessarily carry non-perturbative charges such as magnetic charges or charges in the R-R sector of string theories. The first attempt to calculate string state degeneracy of the corresponding black holes that carry non-perturbative charges is based upon throat limit of a special class of conformal model, called chiral null model [14 [16]. In this description, the throat region is described by a WZNW model with the level determined by NS-NS magnetic charges (carried by black hole solutions). Effectively, the throat region conformal model describing the general class of dyonic solution is the sigma model for perturbative heterotic string with string tension rescaled by magnetic charges.

Another stringy description of dyonic black holes, which is accepted widely and can be applicable to non-BPS black holes as well, was prompted by the realization [17] that non-perturbative R-R charges of type-II string theories can be carried by $D$-branes [18], i.e. boundaries upon which open strings with Dirichlet boundary conditions are constrained to live. With introduction of $D$-branes into string states as carriers of non-perturbative $\mathrm{R}$-R charges, it becomes possible to circumvent 19 24 the previous difficulty of having to count bound states of solitons and perturbative string states when one has to determine the degeneracy of non-perturbative string states that carry R-R charges as well as NS-NS electric charges. In the $D$-brane description of black holes [25,26], microscopic degrees of

freedom of black holes originate from oscillating degrees of freedom of open strings which 
stretch between $D$-branes. Effectively, $D$-brane bound state description of black holes is that of perturbative open strings with central charge rescaled by $\mathrm{R}-\mathrm{R}$ charges or the number of $D$-branes.

In the chiral null model and the $D$-brane descriptions of dyonic black holes, carriers of non-perturbative charges play non-dynamic roles gravitationally and act just as backgrounds in which strings oscillate, effectively playing the role of rescaling string tensions or central charges. (In principle, massive string excitations will modify gravitational field of black holes.) Base upon this observation and 't Hooft's idea [6] that entropy of black holes is nothing but the entropy of fields in thermal equilibrium with black hole environment, Susskind and others 27 30 proposed braneless description of black holes, in an attempt to solve problems associated with stringy statistical interpretation of non-extreme black holes [7]. Namely, in their description of 'braneless' black holes, non-perturbative charges are carried by black holes which act as background gravitational field in which perturbative, NS-NS electric charged strings oscillate. Due to scaling of the time coordinate to the Rindler time nearby the event horizon, string tension and string oscillation levels get scaled 1 by non-perturbative charges. Using this prescription, they were able to reproduce Bekenstein-Hawking entropies of non-rotating black holes in five [four] dimensions with three [four] charges in the BPS and near BPS limit [29],30].

It is one of purposes of this paper to generalize their argument to the case of rotating black holes in string theories. In the comoving coordinates, geometry of rotating black holes approximates to that of Rindler spacetime in the region close to the event horizon, i.e. the throat region. Thereby, the sigma-model description of (rotating) black hole background gets simplified in the comoving frame. We shall build up frameworks for understanding statistical description of rotating black holes in the Rindler spacetime picture and speculate on some of points that we do not have complete understanding of. We believe that precise agreement of the Bekenstein-Hawking entropy and the statistical entropy in this description will require better understanding of subtleties on perturbative strings in the comoving frame.

Main justification for (perturbative) stringy calculation of statistical entropy of black holes hinges on a special property of BPS states that the degeneracy of BPS states is a topological quantity which is independent of coupling constants. Thereby, one can safely calculate the microscopic degeneracy of black holes, which correspond to strong coupling limit, in the weak coupling limit, in which spacetime is Minkowskian and perturbative description of strings is valid. Also, it is supersymmetry (preserved by BPS solutions) that renders quantum corrections under control. On the other hand, it is observed (to the contrary to conventional lore) that even for non-BPS extreme solutions [31,32, near extreme solutions [33 37] and non-extreme solutions [38], D-brane description of black holes reproduces Bekenstein-Hawking entropy correctly. In particular, according to the correspondence principle proposed in Ref. [38], the Bekenstein-Hawking entropies of non-extreme black holes can be reproduced by $D$-brane or perturbative string descriptions when the size of the event horizon is of the order of string scale. The correspondence principle gives rise to statistical

\footnotetext{
${ }^{1}$ Note, the time coordinate is conjugate to energy, since Hamiltonian is the generator for timetranslation.
} 
entropy of non-rotating, non-extreme black holes which is qualitatively in agreement with the Bekenstein-Hawking entropy up to a numerical factor of order one. In this paper, we shall show that the correspondence principle can be applied to special class of electrically charge non-extreme, rotating black holes in heterotic string on tori, as well.

The paper is separated into two parts. In the first part, we shall attempt to formulate Rindler space description of rotating black holes. In the second part, we discuss statistical interpretation of general class of rotating black holes in heterotic string on tori that are constructed in Refs. [13, 39,40] by applying $D$-brane picture and the correspondence principle. In section II, we shall discuss some of global spacetime properties of rotating black holes and derive that in the comoving frame the spacetime approximates to the Rindler spacetime in the region very close to the event horizon. In section III, we discuss statistical description of strings in rotating black hole background and in particular we show that the entropy of strings in the rotating frame is the same as the entropy in the static frame. In section IV, we summarize properties of general classes of non-extreme, rotating black holes in heterotic string on tori constructed in Refs. [13,39,40]. In section $\square$, we discuss the statistical description of such black hole solutions in the picture of $D$-brane and the correspondence principle, and speculate on the Rindler spacetime description of statistical entropy.

\section{SPACETIME PROPERTIES OF ROTATING BLACK HOLES}

In this section we summarize some of properties of rotating black holes necessary in understanding thermodynamics of fields that are in thermal equilibrium with the black hole environment. In addition to the ADM mass and $U(1)$ charges, rotating black holes are characterized by angular momenta. The presence of angular momenta dramatically changes the global spacetime properties of black holes.

In general, spacetime metric for axisymmetric black holes in $D$-dimensional spacetime can be written in the following form in the Boyer-Lindquist coordinates:

$$
d s^{2}=g_{t t} d t^{2}+g_{r r} d r^{2}+2 g_{t \phi_{i}} d t d \phi_{i}+g_{\phi_{i} \phi_{j}} d \phi_{i} d \phi_{j}+g_{\theta \theta} d \theta^{2}+2 g_{\theta \psi_{i}} d \theta d \psi_{i}+g_{\psi_{i} \psi_{j}} d \psi_{i} d \psi_{j},
$$

where $\phi_{i}\left(i=1, \ldots,\left[\frac{D-1}{2}\right]\right)$ correspond to angular coordinates in the $\left[\frac{D-1}{2}\right]$ orthogonal rotational planes and the index $i$ in other angular coordinates $\psi_{i}$ runs from 1 to $\left[\frac{D-4}{2}\right]$. The metric coefficients $g_{\mu \nu}(\mu, \nu=0,1, \ldots, D-1)$ in the Boyer-Lindquist coordinates are independent of $t$ and $\phi_{i}$, manifesting "time-translation invariant" (stationary) and "axially symmetric" spacetime geometry. So, the Killing vectors associated with these symmetries are $\xi_{(t)} \equiv \partial / \partial_{t}$ and $\xi_{\left(\phi_{i}\right)} \equiv \partial / \partial_{\phi_{i}}$.

Due to axially symmetric spacetime, any observer who moves along the worldline of constant $\left(r, \theta, \psi_{i}\right)$ with uniform angular velocity does not notice any change in spacetime geometry. Hence, such an observer can be thought of as "stationary" relative to the local geometry. On the other hand, an observer who moves along the constant $\left(r, \theta, \psi_{i}, \phi_{i}\right)$ worldline, i.e. with zero angular velocity, is also "static" relative to the asymptotic spacetime. Here, the angular velocity $\Omega_{i}\left(i=1, \ldots,\left[\frac{D-1}{2}\right]\right)$ relative to asymptotic rest frame is defined as $\Omega_{i} \equiv \frac{d \phi_{i}}{d t}$. Since an observer cannot move faster than the speed of light, the angular velocity $\Omega_{i}$ of the observer is constrained to take limited values. Namely, the constraint that the 
$D$-velocity $\mathbf{u}=u^{t}\left(\partial / \partial t+\Omega_{i} \partial / \partial \phi_{i}\right)=\frac{\xi_{(t)}+\Omega_{i} \xi_{\phi_{i}}}{\left|\xi_{(t)}+\Omega_{i} \xi_{\phi_{i}}\right|}\left(u^{t} \equiv \frac{d t}{d \tau}\right)$ lies inside the future light cone, i.e. $\mathbf{u} \cdot \mathbf{u}<0$, restricts the angular velocity of stationary observers to be bound by their minimum and maximum values, i.e. $\Omega_{i \min }<\Omega_{i}<\Omega_{i \max }$. The minimum and the maximum values of the angular velocity are explicitly in the following forms:

$$
\Omega_{i \min }=\omega_{i}-\sqrt{\omega_{i}^{2}-g_{t t} / g_{\phi_{i} \phi_{i}}}, \quad \Omega_{i \max }=\omega_{i}+\sqrt{\omega_{i}^{2}-g_{t t} / g_{\phi_{i} \phi_{i}}},
$$

where $\omega \equiv \frac{1}{2}\left(\Omega_{i \min }+\Omega_{i \max }\right)=-g_{t \phi_{i}} / g_{\phi_{i} \phi_{i}}$. Note, $r \Omega_{i \min }=-1$ and $r \Omega_{i \max }=1$ at spatial infinity, corresponding to the speed of light in the Minkowski spacetime.

As observers approach the black hole, $\Omega_{i \text { min }}$ increases and finally becomes zero when $g_{t t}=0$. Therefore, in the region at and inside of the hypersurface defined by $g_{t t}=0$, observers have to rotate (with positive angular velocities), i.e. observers cannot be static relative to the asymptotic rest frame. For this reason, this hypersurface defined by $g_{t t}=0$, i.e. defined as the surface on which the Killing vector $\xi_{t}=\partial / \partial_{t}$ vanishes, is called "static limit". As observers further approach the event horizon (defined as $g^{r r}=0$ surface, on which $r=$ constant surface is null), the range of values that $\Omega_{i}$ can take on narrows down, and finally at the horizon the minimum and maximum values of $\Omega_{i}$ coalesce. This can be seen by the fact that the value of $\omega_{i}=-g_{t \phi_{i}} / g_{\phi_{i} \phi_{i}}$ at the event horizon corresponds to the angular velocity $\Omega_{H i}$ of the event horizon, which is defined by the condition that the Killing vector $\xi \equiv \partial / \partial t+\Omega_{H i} \partial / \partial \phi_{i}$ is null on the horizon. So, at the event horizon, observers cannot be stationary and fall into the black hole through the horizon. Therefore, in the region between the static limit and the event horizon (called "ergosphere") observers are forced to rotate in the direction of the black hole rotation ("dragging of inertial frames").

Due to the "dragging of inertial frames" by the black hole's angular momenta, particles or strings which are in equilibrium with the thermal bath of black hole nearby the event horizon have to rotate with the black hole. Therefore, in order to study thermodynamics of fields in thermal equilibrium with the black hole environment, one has to understand thermodynamics of particles (strings) in the comoving frame which rotates with the black hole. As we will discuss in the following subsection, in the comoving frame which rotates with the angular velocity of the event horizon the spacetime metric in the throat region simplifies to that of the Rindler spacetime. In such frame, analysis of statistical mechanics of strings gets simpler and one can just apply the result of flat-spacetime, non-interacting, perturbative string theory for studying statistical entropy of rotating black holes.

\section{A. Comoving Frame and Rindler Geometry}

We consider the comoving frame which rotates with an angular velocity $\Omega_{i}(i=$ $\left.1, \ldots,\left[\frac{D-1}{2}\right]\right)$ by performing the coordinate transformation $\phi_{i} \rightarrow \phi_{i}^{\prime}=\phi_{i}-\Omega_{i} t$. In this comoving frame, the spacetime metric (1) takes the following form:

$$
\begin{aligned}
d s^{2}=g_{\mu \nu}^{\prime} d x^{\prime \mu} d x^{\prime \nu}= & \left(g_{t t}+2 \Omega_{i} g_{t \phi_{i}}+\Omega_{i} \Omega_{j} g_{\phi_{i} \phi_{j}}\right) d t^{2}+2\left(g_{t \phi_{i}}+\Omega_{j} g_{\phi_{j} \phi_{i}}\right) d t d \phi_{i}^{\prime}+g_{\phi_{i} \phi_{j}} d \phi_{i}^{\prime} d \phi_{j}^{\prime} \\
& +g_{r r} d r^{2}+g_{\theta \theta} d \theta^{2}+2 g_{\theta \psi_{i}} d \theta d \psi_{i}+g_{\psi_{i} \psi_{j}} d \psi_{i} d \psi_{j} .
\end{aligned}
$$

In the comoving frame, one has to restrict the system of particles (strings) to be in the region such that $g_{t t}^{\prime}=g_{t t}+2 \Omega_{i} g_{t \phi_{i}}+\Omega_{i} \Omega_{j} g_{\phi_{i} \phi_{j}}<0$. Namely, in the region defined by $g_{t t}^{\prime}>0$, 
the comoving observer has to move faster than the speed of light. At the boundary surface $g_{t t}^{\prime}=0$, the comoving observer has to move with the speed of light. As we shall see in the following section, the Helmholtz free energy $F$ diverges in the region $g_{t t}^{\prime} \geq 0$, thereby the spacetime region under consideration has to be restricted to $g_{t t}^{\prime}<0$ so that thermodynamic observables are well-defined.

From now on, we consider the Hartle-Hawking vacuum state, which is defined as the system which rotates with the angular velocity of the event horizon and therefore (as we will see) the temperature of the system equals to the Hawking temperature. We are interested in the throat region $\left(r \simeq r_{H}\right)$ of the comoving frame of this system. For this purpose, we consider only the time $t$ and the radial $r$ parts of the spacetime metric, i.e. $d s_{T}^{2}=$ $g_{t t}^{\prime} d t^{2}+g_{r r}^{\prime} d r^{2}$. And we take $\Omega_{i}$ in Eq. (33) to be angular velocity of rotating black hole at the outer horizon, i.e. $\Omega_{i}=\Omega_{H i}$.

Then, the $(t, t)$-component $g_{t t}^{\prime}$ of the metric (3) can be expressed in the following suggestive form in terms of the null Killing vector $\xi=\frac{\partial}{\partial t}+\Omega_{H i} \frac{\partial}{\partial \phi_{i}}$ :

$$
g_{t t}^{\prime}=g_{t t}+2 \Omega_{H i} g_{t \phi_{i}}+\Omega_{H i} \Omega_{H j} g_{\phi_{i} \phi_{j}}=g_{\mu \nu} \xi^{\mu} \xi^{\nu} \equiv-\lambda^{2}
$$

Next, we define new radial coordinate $\rho$ in the following way:

$$
d \rho^{2} \equiv g_{r r} d r^{2} \Rightarrow \rho=\int_{r_{H}}^{r} \sqrt{g_{r r}} d r
$$

Then, the $(t, t)$-component of the metric (3) gets simplified in the near-horizon region as follows:

$$
\begin{aligned}
g_{t t}^{\prime} d t^{2} & =\xi_{\mu} \xi^{\mu} d t^{2}=-\left(\sqrt{-\xi_{\mu} \xi^{\mu}}\right)^{2} d t^{2} \\
& =-\left[\left.\frac{d \sqrt{-\xi_{\mu} \xi^{\mu}}}{d \rho}\right|_{r=r_{H}} \rho\right]^{2} d t^{2}=-\frac{1}{g_{r r}}\left[\left.\frac{d \sqrt{-\xi_{\mu} \xi^{\mu}}}{d r}\right|_{r=r_{H}}\right]^{2} \rho^{2} d t^{2}
\end{aligned}
$$

Note, the surface gravity $\kappa$ at the (outer) event horizon $r=r_{H}$ is defined in terms of the norm $\lambda$ of the null Killing vector $\xi$ as $\kappa^{2} \equiv \lim _{r \rightarrow r_{H}} \nabla_{\mu} \lambda \nabla^{\mu} \lambda$. Therefore, the time-radial parts $d s_{T}^{2}$ of the metric in the vicinity of the event horizon $r=r_{H}$ take the following simplified form 9

$$
d s_{T}^{2}=-\kappa^{2} \rho^{2} d t^{2}+d \rho^{2}
$$

This becomes the Rindler spacetime metric after one rescales the time coordinate $t$ to the Rindler time $\tau \equiv \kappa t$ and performs Euclidean time coordinate transformation. Therefore, we come to the conclusion that in the comoving frame of the Hartle-Hawking vacuum system the throat region approximates to the Rindler spacetime.

\footnotetext{
${ }^{2}$ In can be shown that the other comoving frame metric components $g_{\mu \nu}^{\prime}$ also get simplified in the throat region.
} 


\section{STATISTICAL MECHANICS AND COMOVING FRAME}

We consider the canonical ensemble of statistical system. In this description, the statistical system under consideration is regarded as a macroscopic body which is in thermal equilibrium with some larger "medium" of closed thermal system with a fixed temperature $T$. The larger system behaves like a heat reservoir. The statistical distribution of the canonical ensemble is given by the Gibbs distribution. Therefore, the canonical ensemble is suitable for describing thermodynamics of particles or strings which are in thermal equilibrium with the black hole environment. In the following, we discuss some basic well-known aspects of canonical ensemble for the purpose of setting up notations and discussing statistical mechanics in the comoving frame in general settings (not restricted to just some special class of rotating black holes.)

The basic quantity of the canonical ensemble is the canonical partition function which is defined as a functions of a variable $\beta$ :

$$
Z(\beta) \equiv \sum_{\alpha} e^{-\beta E_{\alpha}}=\operatorname{Tr} \exp (-\beta H)
$$

where the trace is over the states $\alpha$ with the energy $E_{\alpha}$ and $H$ denotes the Hamiltonian of the smaller subsystem. Classically, the partition function $Z(\beta)$ corresponds to the volume in the phase space occupied by the canonical ensemble. Here, $\beta$ is a real constant interpreted as the inverse temperature of the larger system with which the statistical ensemble under consideration is in thermal equilibrium.

One can re-express the partition function (8) in the following way:

$$
\begin{aligned}
Z(\beta) & =\sum_{\alpha} e^{-\beta E_{\alpha}}=\sum_{\alpha} \int_{0}^{\infty} d E \delta\left(E-E_{\alpha}\right) e^{-\beta E} \\
& =\int_{0}^{\infty} d E\left[\sum_{\alpha} \delta\left(E-E_{\alpha}\right)\right] e^{-\beta E}=\int_{0}^{\infty} d E g(E) e^{-\beta E}
\end{aligned}
$$

where $g(E)=\sum_{\alpha} \delta\left(E-E_{\alpha}\right)$ is the total density of states, i.e. $g(E) d E$ is the total number of energy eigenstates $\alpha$ of the system in the energy range from $E$ to $E+d E$. Note, from Eq.(9) one can see that the partition function $Z(\beta)$ is nothing but the Laplace transform of the total density of states $g(E)$. So, alternatively one can obtain $g(E)$ from the partition function through the inverse Laplace transformation $g(E)=\int_{\varepsilon-i \infty}^{\varepsilon+i \infty} \frac{d \beta}{2 \pi i} e^{\beta E} Z(\beta)$, where the contour (defined by a real number $\varepsilon$ ) is chosen to be to the right of all the singularities of $Z(\beta)$ in the complex $\beta$ plane.

The partition function $Z(\beta)$ is related to the Helmholtz free energy $F$ in the following way:

$$
F=-\frac{1}{\beta} \ln Z=-T \ln \sum_{\alpha} e^{-E_{\alpha} / T}
$$

where $\beta=1 / T$. This follows from the following definition of the entropy $S$ :

$$
S \equiv-\sum_{\alpha} \rho_{\alpha} \ln \rho_{\alpha}=-\operatorname{Tr}(\rho \ln \rho)
$$


where $\rho_{\alpha}=e^{-E_{\alpha} / T} /\left(\sum_{\alpha} e^{-E_{\alpha} / T}\right)$ is the "Gibbs distribution" or "canonical distribution", and from the definition of the free energy $F \equiv E-T S$. (Here, the energy $E$ is taken as the mean energy $\bar{E} \equiv \sum_{\alpha} E_{\alpha} \rho_{\alpha}$.) So, alternatively entropy $S$ can be obtained from the Helmholtz free energy $F$ as

$$
S=\beta^{2} \frac{\partial F}{\partial \beta}=-\frac{\partial F}{\partial T}
$$

In terms of the total energy density of the state $g(E)$, the free energy takes the following form:

$$
F=\frac{1}{\beta} \int_{0}^{\infty} d E g(E) \ln \left(1-e^{-\beta E}\right)
$$

For the canonical ensemble of non-interacting particles (strings) that rotate with constant azimuthal angular velocity $\Omega_{i}$, the partition function $Z(\beta)$ in Eq.(8) gets modified to

$$
Z=\sum_{\alpha} e^{-\beta\left(E_{\alpha}-\Omega_{i} J_{i \alpha}\right)}
$$

where $E_{\alpha}$ is the energy in the rest frame and $J_{i}$ are the $\phi_{i}$-components of angular momentum of the particles (strings). This is a special example of the case in which the statistical system has the set of conserved quantities $Q$ other than energy $E$. Examples on the conserved quantities $Q$ are angular momentum, string winding number, electric charges, etc.. (For this case, the conserved quantity $Q$ corresponds to the angular momentum $\vec{J}$.) In general, the total energy density of the system with set $Q$ of conserved quantities is given by

$$
g(E, Q)=\sum_{\alpha} \delta\left(E-E_{\alpha}\right) \delta_{Q, Q_{\alpha}}
$$

So, for example, the partition function (14) can be derived from the total energy density given by (15) with $Q=\vec{J}$ by following the similar procedure as Eq.(9). The final expression is of the form:

$$
Z(\beta)=\sum_{J_{i}} \int_{0}^{\infty} d E g\left(E, J_{i}\right) e^{-\beta\left(E-\Omega_{i} J_{i}\right)}
$$

where the sum is over the azimuthal quantum numbers of the angular momentum $\vec{J}$. And the Helmholtz free energy is, therefore, given by

$$
F=\frac{1}{\beta} \sum_{J_{i}} \int_{0}^{\infty} d E g\left(E, J_{i}\right) \ln \left(1-e^{-\beta\left(E-\Omega_{i} J_{i}\right)}\right) .
$$

In the following, we show that entropy of rotating systems takes the same form whether it is calculated in the comoving-frame or in the rest-frame. Therefore, one can conveniently calculate the entropy in the comoving frame of the Hartle-Hawking vacuum system, in which the spacetime metric takes the simple Rindler spacetime form. This is consistent with the fact that the entropy measures the degeneracy of the number of indistinguishable microscopic states, which should be independent of the coordinate frame of an observer. 
We consider particles (strings) that rotate with angular velocity $\Omega_{i}$. In the rest frame, the partition function for such rotating canonical ensemble is given by Eq.(14) or alternatively, in terms of the total energy density, by Eq.(16), and the Helmholtz free energy is given by Eq.(17). On the other hand, in the comoving frame (that rotates with the particles or the strings) the angular velocity of the system of particles or strings is zero. So, the partition function is of the following form

$$
Z(\beta)=\sum_{\alpha} e^{-\beta E_{\alpha}^{\prime}}=\int_{0}^{\infty} d E^{\prime} g\left(E^{\prime}\right) e^{-\beta E^{\prime}},
$$

where $E^{\prime}$ is the energy in the comoving frame. And correspondingly the Helmholtz free energy is given by

$$
F=\frac{1}{\beta} \int_{0}^{\infty} d E^{\prime} g\left(E^{\prime}\right) \ln \left(1-e^{-\beta E^{\prime}}\right)
$$

Note, in the rest frame the requirement that the free energy $F$ in Eq.(17) takes finite real value restricts the interval of the integration over $E$ to be $E>\Omega_{i} J_{i}$. Namely, when $E=\Omega_{i} J_{i}$ the integrand of Eq.(17) diverges and when $0<E<\Omega_{i} J_{i}$ the integrand takes a complex value. For the case of particles (strings) in the background of rotating black holes, such restriction of the integration interval corresponds to the condition that the speed of particles (strings) has to be less than the speed of light, i.e. $g_{t t}^{\prime}=g_{t t}+2 \Omega_{i} g_{t \phi_{i}}+\Omega_{i} \Omega_{j} g_{\phi_{i} \phi_{j}}<0$. Therefore, the Helmholtz free energy $F$ in Eq.(17) becomes

$$
\begin{aligned}
F & =\frac{1}{\beta} \sum_{J_{i}} \int_{\Omega_{i} J_{i}}^{\infty} d E g\left(E, J_{i}\right) \ln \left(1-e^{-\beta\left(E-\Omega_{i} J_{i}\right)}\right) \\
& =\frac{1}{\beta} \int_{0}^{\infty} d E \sum_{J_{i}} g\left(E+\Omega_{i} J_{i}, J_{i}\right) \ln \left(1-e^{-\beta E}\right) \\
& =-\int_{0}^{\infty} d E \frac{1}{e^{\beta E}-1} \sum_{J_{i}} \Gamma\left(E+\Omega_{i} J_{i}, J_{i}\right),
\end{aligned}
$$

where in the second equality the change of integration variable $E \rightarrow E-\Omega_{i} J_{i}$ is performed and in the third equality we have integrated by parts. Here, $\Gamma\left(E, J_{i}\right)$ is the total number of states with energy less than $E$, given that the angular momentum is $J_{i}$. It can be shown that $\sum_{J_{i}} \Gamma\left(E+\Omega_{i} J_{i}, J_{i}\right)=\Gamma(E)$. Therefore, the Helmholtz free energy (17) in the rest-frame takes the same form as the free energy (19) in the comoving frame. Similarly, one can show that the partition functions $Z(\beta)$ in the rest-frame and the comoving frame have the same

form. Consequently, entropy $S=\beta^{2} \frac{\partial F}{\partial \beta}$ takes the same values in the comoving and the rest frames.

\section{A. Thermodynamics of the Rindler Spacetime}

We showed in section IIA that in the comoving frame which rotates with the angular velocity of the event horizon the spacetime metric for a rotating black hole in the BoyerLindquist coordinates approximates to the metric of Rindler spacetime in the throat region. Therefore, as long as we are interested in the throat region in the comoving frame, the 
statistical analysis (based on the string sigma model with the target space background given by the rotating black hole solution) becomes remarkably simpler. In the following, we discuss some aspects of thermodynamics of the Rindler spacetime.

The Rindler spacetime is spacetime of uniformly accelerating observer, called Rindler observer, in the Minkowski spacetime. The Rindler spacetime covers only a quadrant of Minkowski spacetime, defined by the wedge $x>|t|$, where $x$ is in the direction of uniform acceleration. Since the trajectory of the Rindler observer's motion approaches the null rays $u \equiv t-x=0$ and $v \equiv t+x=0$ asymptotically as $t \rightarrow \pm \infty$, these rays act as event horizons. The metric of the Rindler spacetime has the following form:

$$
d s^{2}=\rho^{2} d \tau^{2}+d \rho^{2}+d \vec{x}_{\perp}^{2},
$$

where $\tau$ is the (Euclidean) Rindler time and $\vec{x}_{\perp}$ is the coordinates of the space parallel to the event horizon located at the surface $\rho=0$.

After the time scale transformation followed by the Euclidean time coordinate transformation, the metric of the throat region of rotating black holes in the comoving frame of Hartle-Hawking vacuum becomes the metric of the Rindler spacetime. In the following, we discuss the relationship between thermodynamic observerbles in the comoving frame and the Rindler frame, closely following Refs. [27,29, 30. Note, the rotation of particles (strings) which are in equilibrium with rotating black holes is not rigid, but have locally different angular velocities. Therefore, there is no globally static coordinates for particles (strings). But, for the simplicity of calculation, in the following we assume that particles (strings) uniformly rotate with the angular velocity of the event horizon, or in other words the angular momenta of particles (strings) are zero in the comoving frame since the coordinate frame rotates with them. This approximation can be justified since we are assuming that the contribution to the statistical entropy is from strings which are nearby the event horizon and, therefore, are somehow entangled with the event horizon. Another setback of the comoving frame description of black hole entropy (in general, without restriction to the throat region) is that the fields in the region beyond the 'velocity of light surface' have to be excluded, since the comoving observer has to move faster than the speed of light in this region or equivalently (as we discussed in section [II) thermodynamic observables in this region are not well-defined. This velocity of light surface approaches horizon as the angular momenta of black holes become large. For these reasons, we speculate that the comoving frame description of black hole entropy becomes accurate for small values of angular momenta.

First, we consider the Rindler spacetime with metric given by Eq.(21). The partition function for canonical ensemble of fields is given by Eq.(8) with the Hamiltonian $H_{R}$ being the generator of the $\tau$-translations, i.e. $H_{R}=\frac{\partial}{\partial \tau}$, which satisfies the commutation relation $\left[H_{R}, \tau\right]=1$. One can apply the usual thermodynamic relations to obtain other thermodynamic observables of the canonical ensemble in the Rindler spacetime. In particular, the temperature in the Rindler spacetime (with which the canonical ensemble of fields is in thermal equilibrium) is always $T_{R}=\frac{1}{2 \pi}$, which follows from the requirement of the absence of the conical singularity in the $(\tau, \rho)$-plane. The first law of thermodynamics of canonical ensemble of fields which are in thermal equilibrium with the Rindler spacetime, therefore, takes the following form:

$$
d E_{R}=\frac{1}{2 \pi} d S_{R}
$$


where $E_{R}$ and $S_{R}$ are respectively energy and entropy of fields in the Rindler spacetime.

Second, for the spacetime of throat region in the comoving frame with metric given by Eq.(7), the similar analysis can be done. The Hamiltonian $H$ for fields is the generator for the $t$-translation, i.e. $H=\frac{\partial}{\partial t}$, which satisfies the commutation relation $[H, t]=1$. The temperature of this spacetime is $T=\frac{\kappa}{2 \pi}$, where $\kappa$ is the surface gravity at the event horizon of the rotating black holes. The first law of thermodynamics of canonical ensemble of fields which are in thermal equilibrium with this spacetime is, therefore, given by

$$
d E=\frac{\kappa}{2 \pi} d S
$$

where $E$ and $S$ are respectively the energy and the entropy of fields in the comoving frame.

Note, the time coordinates $\tau$ and $t$ of both the spacetimes are related by the rescaling $\tau=\kappa t$ (up to imaginary number $i$ ). So, the energy $E$ of fields is related to the Rindler energy $E_{R}$ as $d E_{R}=\frac{1}{\kappa} d E$. (This can be understood from the relationship $1=\left[E_{R}, \tau\right]=$ $\kappa\left[E_{R}, t\right]=\kappa \frac{\partial E_{R}}{\partial E}$.) Therefore, it follows from (23) that entropy $S$ of fields in the comoving frame is related to the Rindler energy $E_{R}$ in the following way

$$
S=2 \pi E_{R}
$$

\section{B. Thermodynamics of Strings}

In this section, we discuss thermodynamics of non-interacting string gas in perturbative string theory in flat target space background. Statistical properties of superstring gas are obtained by calculating energy level densities and using equipartition. These are already well-known, but we will discuss some of aspects for the sake of setting up notations and preparing for discussions in the later sections.

In general, for the purpose of calculating energy level density of strings one introduces the generating function

$$
\Pi(q) \equiv \sum_{n=1}^{\infty} d(n) q^{n}
$$

where $d(n)$ is the degeneracy of states at the oscillator level $N=n$. For bosonic and fermionic $m$-oscillators, the corresponding generating functions $\pi_{m}^{b}(q)$ and $\pi_{m}^{f}(q)$ take the following forms:

$$
\begin{aligned}
& \pi_{m}^{b}(q)=1+q^{m}+q^{2 m}+\cdots=\frac{1}{1-q^{m}}, \\
& \pi_{m}^{f}(q)=1+q^{m} .
\end{aligned}
$$

Each oscillator (labeled by $m$ ) contributes to the generating function multiplicatively. Therefore, the generating function has the following structure:

$$
\Pi(q)=\Pi_{B}(q) \Pi_{F}(q),
$$


where $\Pi_{B}(q)$ and $\Pi_{F}(q)$ are, respectively, bosonic and fermionic contributions to the generating function, and take the following forms:

$$
\Pi_{B}(q)=\prod_{m=1}^{\infty}\left(1-q^{m}\right)^{-n_{b}}, \quad \Pi_{F}(q)=\prod_{m=1}^{\infty}\left(1+q^{m}\right)^{n_{f}} .
$$

Here, $n_{b}$ and $n_{f}$ depend on the degrees of freedom of bosonic and fermionic coordinates. For heterotic strings, the bosonic factor $\Pi_{B}(q)$ has an additional multiplicative factor originated from states from the compactification on the sixteen-dimensional, self-dual, even, integral lattice.

In order to obtain the degeneracy $d(n)$ of string states at the $n$-th oscillator level from the generating function $\Pi(q)$, one performs the following contour integral:

$$
d(n)=\frac{1}{2 \pi i} \int_{C} \frac{d z}{z} \frac{1}{z^{n}} \Pi(z)
$$

By applying the saddle-point approximation of the contour integral, one obtains the following large- $n$ approximation for $d(n)$ :

$$
d(n) \simeq n^{-(D+1) / 4} e^{\sqrt{n / \alpha^{\prime}} \beta_{H}},
$$

where $D$ is the dimension of spacetime in which strings live, i.e. $D=10[D=26]$ for superstrings [bosonic strings]. Here, the parameter $\beta_{H}$ depends on fermionic spectrum: $\beta_{H} / \sqrt{\alpha^{\prime}}=\pi \sqrt{D-2}$ with fermions and $\beta_{H} / \sqrt{\alpha^{\prime}}=\pi \sqrt{\frac{2}{3}(D-2)}$ without fermions. Then, the density of states $\rho(m)$ in mass $m$ has the following general form that resembles the density of hadron level obtained from, for example, dual models:

$$
\rho(m) \simeq m d(m) \simeq c m^{-a} \exp (b m)
$$

where for heterotic strings $a=10$ and $b=(2+\sqrt{2}) \pi \sqrt{\alpha^{\prime}}$, for type-I superstrings $a=\frac{9}{2}$ and $b=\pi \sqrt{8 \alpha^{\prime}}$, and for closed superstrings $a=10$ and $b=\pi \sqrt{8 \alpha^{\prime}}$.

From the density of states $\rho(m)$, one obtains the partition function $Z(V, T)$ for canonical ensemble of (relativistic) superstrings enclosed in a box of volume $V$ at a temperature $T$ by following the procedure developed in Refs. 41 43]:

$$
\begin{aligned}
\ln Z & =\frac{V}{(2 \pi)^{9}} \int d m \rho(m) \int d^{9} k \ln \frac{1+e^{-\beta \sqrt{k^{2}+m^{2}}}}{1-e^{-\beta \sqrt{k^{2}+m^{2}}}} \\
& \simeq V \sum_{n=0}^{\infty}\left(\frac{1}{2 n+1}\right)^{5} \int \rho(m) m^{-a+5} K_{5}[(2 n+1) \beta m] e^{b m} d m \\
& \simeq\left(\frac{T T_{0}}{T_{0}-T}\right)^{-a+11 / 2} \Gamma\left[-a+\frac{11}{2}, \eta\left(\frac{T_{0}-T}{T T_{0}}\right)\right],
\end{aligned}
$$

where $\beta=1 / T, K_{5}$ is the modified Bessel function with the asymptotic expansion of the form $K_{5}(z) \simeq\left(\frac{\pi}{2 z}\right)^{1 / 2} e^{-z}\left(1+\frac{15}{8 z}+\cdots\right), \Gamma(a, x)$ is the incomplete gamma function, and $T_{0}=1 / b$. Note, the partition function $Z$ diverges for $T>T_{0}$, implying that $T_{0}$ is the maximum temperature for thermodynamic equilibrium, i.e. the Hagedorn temperature [42]. 
From the above expression for the partition function, one can calculate the thermodynamic observables: $P=T \partial \ln Z / \partial V, C_{V}=d\langle E\rangle / d T$, and $\langle E\rangle=T^{2} \partial \ln Z / \partial T$, etc..

The statistical entropy $S_{\text {stat }}$ of gas of strings is defined by the logarithm of the degeneracy of string states at oscillator levels $\left(N_{R}, N_{L}\right)$ :

$$
S_{\text {stat }}=\ln d\left(N_{R}, N_{L}\right) \simeq 2 \pi\left(\sqrt{\frac{c_{R}}{6}} \sqrt{N_{R}}+\sqrt{\frac{c_{L}}{6}} \sqrt{N_{L}}\right)=S_{R}+S_{L},
$$

where $c_{R, L}=n_{R, L}^{b}+\frac{1}{2} n_{R, L}^{f}$ are the central charges, which are determined by the bosonic $n_{R, L}^{b}$ and the fermionic $n_{R, L}^{f}$ degrees of freedom in the right-moving and the left-moving sectors, and $N_{R}\left[N_{L}\right]$ is the right [left] moving oscillator level. Here, $S_{R}$ and $S_{L}$ are respectively the entropies of the left-movers and the right-movers. Namely, since we assume that string gas is non-interacting, the total entropy $S_{\text {stat }}$ is expressed as the sum of contributions of two mutually non-interacting sectors, i.e. the right-moving sector and the right-moving sector.

\section{String Thermodynamics in Rindler Spacetime}

We discuss the statistical mechanics of strings in the Rindler spacetime or the comoving frame. In general, statistical mechanics of strings in rotating black hole background is involved due to non-trivially complicated target space manifold for string sigma model. However, in the picture of black hole entropy, proposed by 't Hooft [6] and generalized by Susskind [7] to the case of string theories, i.e. entropy of black holes is nothing but entropy of strings nearby horizon which are in thermal equilibrium with black holes, the analysis of string sigma model gets simplified. In the throat region of the comoving frame that rotates with rotating black holes, the spacetime gets approximated to the Rindler spacetime. Therefore, one can apply the relatively well-understood statistical mechanics of perturbative strings in the target space background of the Rindler spacetime for studying statistical entropy of rotating black holes.

In the past, analysis of statistical mechanics of non-extreme black holes in string theories had a setback due to mismatch in dependence on mass of the densities of states of black holes and the string level density. Namely, whereas the number of states in non-extreme black holes grows with the ADM mass $M_{A D M}$ like $\sim e^{M_{A D M}^{2}}$, the string state level density grows with the string state mass $M_{s t r}$ as $\sim e^{M_{s t r}}$. So, if one takes this fact literally, then for large enough mass the quantum states of non-extreme black holes are much denser than those of a perturbative strings with the same mass. In Ref. [7], Susskind proposed to identify the square of the mass of non-extreme black hole with the mass of string states, i.e. $M_{A D M}^{2}=M_{s t r}$, in order to remedy the mismatch. Susskind justified such an identification by postulating that this is due to some unknown quantum correction of black hole mass. In the picture of Rindler spacetime description [27], such mismatch can be understood as being originated from the blueshift of the energy of the string oscillations in the gravitational field of black holes ?. This blueshift of the string oscillation energy causes the rescaling of string

\footnotetext{
${ }^{3}$ In the picture of "correspondence principle" [38], which will be discussed in the later section,
} 
tension (redshieft of the string tension) and string oscillation levels from its free string value in the Minkowski spacetime. Taking this effect into account, it is shown [29,30 that the Bekenstein-Hawking entropy of non-extreme, non-rotating black holes can be reproduced by counting degeneracy of perturbative string states, only. It is one of the purposes of this paper to generalize this picture to the case of rotating black holes.

In the Rindler spacetime description of black holes proposed in Ref. [27], black hole configuration is divided into two sectors: the first sector carrying (perturbative) NS-NS electric charges and the second sector carrying non-perturbative charges, i.e. NS-NS magnetic charges and R-R charges. In the weak string coupling limit, NS-NS electric charges are carried by perturbative string states and non-perturbative charges are carried by black holes, which act as backgrounds in which strings oscillate. In this description, back-reaction of massive string states on the gravitational field of non-perturbative-charge-carrying black holes is neglected. (Therefore, for example the mass of the whole black hole configuration is just a sum of the mass of perturbative string state and the ADM mass of non-perturbativecharge-carrying background black hole.) Note also that even in the weak string coupling limit the carrier of non-perturbative charges remain as black holes, rather than becoming, for example, the bound state of $D$-branes. However, due to the non-trivial background geometry the string tension and string oscillator levels get scaled relative to the free string value.

Although the Rindler spacetime is a flat spacetime, the quantization of strings is nontrivial due to the event horizon and the fact that strings are extended objects. The quantum theory of strings in the Rindler spacetime (or the uniformly accelerating frame in the Minkowski spacetime) is similar to the case of point-like particles except some modifications due to the fact that strings are extended objects. In the following, we discuss some of aspects of string theories in the Rindler spacetime in relation to statistical entropy of rotating black holes.

The coordinates $X^{A}$ of strings in a uniformly accelerating frame of the flat spacetime is related to the coordinates $\hat{X}^{A}$ of the inertial frame through the following transformations:

$$
\begin{aligned}
\hat{X}^{1}-\hat{X}^{0} & =e^{\alpha\left(X^{1}-X^{0}\right)}, \\
\hat{X}^{1}+\hat{X}^{0} & =e^{\alpha\left(X^{1}+X^{0}\right)}, \\
\hat{X}^{i} & =X^{i}, \quad 2 \leq i \leq D-1,
\end{aligned}
$$

where a constant $\alpha$ defines the proper acceleration of the Rindler observers and $D$ is the spacetime dimensions of the theory. Therefore, in a uniformly accelerating frame the target

the mass of black holes $M_{A D M} \sim r_{H} / 2 G_{N}$ cannot always be equal to the mass of string states $M_{s t r} \sim \sqrt{N / \alpha^{\prime}}$, for any values of string coupling $g_{s}$. Namely, since the gravitational constant $G_{N}$ depends on the string coupling $g_{s}$ and $\alpha^{\prime}$ as $G_{N} \sim g_{s}^{2} \alpha^{\prime}$, the ratio of the two masses $M_{s t r} / M_{B P S}$ is a function of string coupling $g_{s}$, and becomes one only at the particular value of string coupling $g_{s}$. At this critical value of string coupling $g_{s}$, the size of horizon $r_{H}$ becomes of the order of string scale $l_{s}=\sqrt{\alpha^{\prime}}$, i.e. $r_{H} \sim l_{s}$, meaning that strings begin to form black holes due to strong gravitational field. And at this critical point, the density of quantum states of black holes agrees with that of perturbative string states. 
space metric $G_{A B}(X)$ takes the following form

$$
G_{A B}(X) d X^{A} d X^{B}=\alpha^{2} e^{2 \alpha X^{1}}\left[\left(d X^{1}\right)^{2}-\left(d X^{0}\right)^{2}\right]+\left(d X^{i}\right)^{2} .
$$

Defining the light-cone variables

$$
U \equiv X^{1}-X^{0}, \quad V \equiv X^{1}+X^{0}, \quad x_{ \pm}=\sigma \pm \tau,
$$

where $(\tau, \sigma)$ are the worldsheet coordinates, one obtains the following sigma-model action in the uniformly accelerating frame

$$
\begin{aligned}
S_{\text {string }} & =\frac{1}{2 \pi \alpha^{\prime}} \int d \sigma d \tau \sqrt{g} g^{\alpha \beta} G_{A B}(X) \partial_{\alpha} X^{A} \partial_{\beta} X^{B} \\
& =\frac{1}{2 \pi \alpha^{\prime}} \int d \sigma d \tau\left[\alpha^{2} e^{\alpha(U+V)} \partial_{\beta} U \partial^{\beta} V+\partial_{\beta} X^{i} \partial^{\beta} X^{i}\right],
\end{aligned}
$$

where the worldsheet metric $g_{\alpha \beta}$ is in the conformal gauge, i.e. $g=\rho(\sigma, \tau) \operatorname{diag}(-1,1)$. The bosonic coordinates $X^{A}(\sigma, \tau)$ satisfy the usual string boundary conditions, e.g. $X^{A}(\sigma+$ $2 \pi, \tau)=X^{A}(\sigma, \tau)$ for closed strings.

One can understand the coordinate transformations (34) in terms of symmetries of the string sigma model and the basic properties of quantum field theory in the following way.

Due to the invariance of the string sigma model action under the worldsheet-coordinate reparametrization $\xi^{\alpha} \rightarrow \xi^{\alpha}+\epsilon^{\alpha}(\xi)$, where $\xi^{\alpha} \equiv(\tau, \sigma)$ are the worldsheet coordinates of strings, one can bring the worldsheet metric $g_{\alpha \beta}(\xi)$ into the conformal form mentioned above. The conformal gauge fixed sigma-model action still has the world sheet coordinate reparametrization invariance of the following form:

$$
x_{+}=f\left(x_{+}^{\prime}\right), \quad x_{-}=g\left(x_{-}^{\prime}\right) .
$$

Note, in quantum field theories it is well-known that Fock spaces built from the canonical states are different for different coordinate basis. Namely, the vacua defined by positive frequency states for a given time coordinate are not vacuum states of another coordinate basis with different time-coordinate. Specializing to the case of string theories, the positive frequency modes defined in the worldsheet reparametrization transformed basis $\xi^{\prime \alpha}=\left(\tau^{\prime}, \sigma^{\prime}\right)$ (Cf. see Eq.(38)) are not positive frequency modes with respect to the target space timecoordinate $X^{0}$. In order to construct well-defined positive frequency modes (which are required for defining particle states in a given reference frame) in the new worldsheet coordinates $\xi^{\prime}=\left(\tau^{\prime}, \sigma^{\prime}\right)$, one has to perform the following target space coordinate transformations:

$$
\begin{aligned}
X_{1}-X_{0}+\epsilon & =f\left(X_{1}^{\prime}-X_{0}^{\prime}\right) \\
X_{1}+X_{0}+\epsilon & =g\left(X_{1}^{\prime}+X_{0}^{\prime}\right) \\
X^{i} & =X^{\prime i}, \quad 2 \leq i \leq D-1 .
\end{aligned}
$$

New frame parameterized by the coordinates $X^{\prime A}$ corresponds to an accelerated reference frame with acceleration $a=\left[f^{\prime} g^{\prime}\right]^{-1 / 2} \partial_{X_{1}^{\prime}}\left[\ln \left(f^{\prime} g^{\prime}\right)\right]$. Now, the positive frequency modes with respect to the worldsheet time coordinate $\tau^{\prime}$ correspond to positive frequency modes with respect to the new target space time-coordinate $X^{\prime 0}$. Here, a constant $\epsilon$ is related to the ultra-violet cut-off $H$ (through $\epsilon \simeq e^{-\alpha H}$ ) on the negative Rindler coordinate $X^{1}$ (namely, 
$\left.X^{1} \geq-H\right)$, which regularizes the divergence in the free energy and entropy of quantum fields due to the existence of the Rindler horizon. This regulator shifts the Rindler horizon at $x^{1}=\left|x^{0}\right|$ to the hyperbola defined by $\left(x^{1}\right)^{2}-\left(x^{0}\right)^{2}=e^{-2 \alpha H} \simeq \epsilon^{2}$.

In particular, the particular case with $f=g=\exp \left(\alpha U^{\prime}\right)$ corresponds to the boost coordinate transformations (34) between an accelerating frame and the inertial frame, with the ultra-violet cut-off taken into account. (Note, in the notation of the transformations (38) and (39), the inertial frame coordinates are $\xi^{\alpha}=(\tau, \sigma)$ and $X^{A}$ without primes, and the accelerating frame coordinates are $\xi^{\prime \alpha}=\left(\tau^{\prime}, \sigma^{\prime}\right)$ and $X^{\prime A}$ with primes.) Therefore, one can think of the boost transformation (34) in the target spacetime as a subset of worldsheet coordinate reparametrization symmetry. As a consequence, the string state spectrum of strings in the Rindler spacetime (or comoving frame) has to be in one-to-one correspondence with that of strings in the Minkowski spacetime (or inertial frame). Namely, the mass spectra and level densities in both the frames have to have the same structures since they are related by one of symmetries of string sigma-model, i.e. worldsheet reparametrization invariance. Equivalently, the boost transformations (34) in the target spacetime have to be accompanied by the appropriate worldsheet reparametrization transformation, so that the positive frequency modes have well-defined meaning. To put it another way, in order to define the light-cone gauge, in which the target space time-coordinate is proportional to the worldsheet time-coordinate with the proportionality constant given by the zero mode of the string center-of-mass frame momentum, one has to simultaneously perform worldsheet coordinate transformations.

In terms of new worldsheet coordinates $\xi^{\prime \alpha}=\left(\tau^{\prime}, \sigma^{\prime}\right)$, the periodicity of the target space coordinates of closed strings is modified to $\Pi_{\epsilon}=\frac{1}{\alpha} \ln \left(\frac{2 \pi}{\epsilon}+1\right)$. So, in particular one can see that the ultra-violet cut-off was needed in order to insure the finite period for closed strings. The frequencies of the Rindler frame basis modes differ from those of the inertial frame modes by a factor $\frac{2 \pi}{\Pi_{e}}$. Also, the momentum zero modes of the bosonic coordinate expansions in terms of the Rindler frame orthonormal basis are differ from those in terms of the inertial frame orthonormal basis by the factor of $\frac{2 \pi}{\Pi_{e}}$. As a consequence, the string oscillation levels in the Rindler frame get rescaled relative to their Minkowski spacetime values. This is related to the fact that the string length in the Rindler frame is different from its length in the inertial frame. This is a reminiscence of the rescaling of the effective length of open strings when $D$-branes are present [4] in the $D$-brane picture of black holes.

The creation operators and the annihilation operators of the inertial frame and the Rindler frame are related by a Bogoliubov transformation. Just as in the case of point-like particle quantum field theories, the expectation value of the Rindler frame number operator with respect to the inertial frame vacuum state has characteristic of Planckian spectrum at temperature $T=\frac{\alpha}{2 \pi}$, with an additional correction term of the Rayleigh-Jeans type due to the fact that string has a finite length. In other words, the uniformly accelerating observer (the Rindler observer) in the Minkowski vacuum state will detect thermal radiation of strings at temperature $T=\frac{\alpha}{2 \pi}$.

The mass formulae of string states in the inertial frame and the Rindler frame have different forms, but have the same eigenvalues. This is in accordance with the expectation that the mass of string states should not change since the sum of the mass of perturbative string states and the ADM mass of background black hole has to remain equal to the 
ADM mass of the whole black hole configuration [27,29,30]. Furthermore, the mass spectra as measured in the accelerating frame and in the inertial frame have the same structure. Therefore, the thermodynamic relations of strings in the Rindler frame (i.e. the throat region in the comoving frame of rotating black holes) have same form as those in the inertial frame (i.e. the Minkowski spacetime), except that the string oscillation levels and string tension are rescaled.

In the following, we discuss some of thermodynamic relations of string gas in the Rindler frame. In general, we believe that the argument on string thermodynamics is slightly different from the one in section IIIA, which we think applies rather to point-like particle field theory, as oppose to the formalism followed in Refs. [27,29, 30]. Namely, unlike particles strings have the left-moving and the right-moving degrees of freedoms. Due to the main assumption of this paper that the gas of strings is non-interacting, the left-moving and the right-moving sectors of the gas of strings form two separate thermodynamic systems with different equilibrium temperatures $T_{L}$ and $T_{R}$, respectively. The total entropy $S$ of the whole system is therefore the sum of the entropy $S_{L}$ of the left-moving sector and the entropy $S_{R}$ of the right-moving sector, i.e. $S=S_{L}+S_{R}$. Also, the total energy $E$ is splited into the left-moving and the right-moving pieces, i.e. $E=E_{L}+E_{R}$. Therefore, the first law of thermodynamics of string gas has to be considered separately for the left-movers and the right-movers in the following way:

$$
d S_{L}=\frac{1}{T_{L}} d E_{L}, \quad d S_{R}=\frac{1}{T_{R}} d E_{R} .
$$

The temperatures $T_{L}$ and $T_{R}$ of the left-movers and the right-movers are related to the Hagedorn temperature $T$ at which weakly coupled strings radiate in the following way:

$$
\frac{1}{T}=\frac{d S}{d E}=\frac{d\left(S_{L}+S_{R}\right)}{d E}=2\left(\frac{d S_{L}}{d E_{L}}+\frac{d S_{R}}{d E_{R}}\right)=2\left(\frac{1}{T_{L}}+\frac{1}{T_{R}}\right),
$$

where we made use of the fact that $\delta E=\delta E_{L}+\delta E_{R}=2 \delta E_{L}=2 \delta E_{R}$ when the total momentum is fixed (i.e. $\delta P=\delta E_{L}-\delta E_{R}=0$ ) [45].

As it is pointed out in section [IIA, due to the scaling of the time-coordinate $t$ to the Rindler space time-coordinate $\tau$ by the factor of the surface gravity $\kappa=2 \pi T_{H}$ at the event horizon of the background black hole, i.e. $\tau=\kappa t$, the energy of the left-moving (the rightmoving) strings in the Rindler frame $E_{\text {Rindler } L, R}$ is related to the energy of strings in the comoving frame $E_{L, R}$ as $d E_{\text {Rindler } L, R}=\frac{1}{\kappa} d E_{L, R}$. So, from the first laws of thermodynamics of the left-moving and the right-moving sectors of strings in Eq.(40), one has the following relations of the total energies of the gas of the left-moving and the right-moving strings in the Rindler frame $E_{\text {Rindler } L, R}$ to the entropies of the left-moving and the right-moving strings $S_{L, R}$ :

$$
d S_{L}=\frac{\kappa}{T_{L}} d E_{\text {Rindler } L}, \quad d S_{R}=\frac{\kappa}{T_{R}} d E_{\text {Rindler } R} .
$$

\section{ROTATING BLACK HOLES IN HETEROTIC STRING ON TORI}

In this section, we summarize properties of the general class of charged, rotating black hole solutions in heterotic string on tori which are constructed in Refs. [13, 39, 40]. 


\section{A. Electrically Charged Rotating Black Holes in Toroidally Compactified Heterotic Strings in $D$-Dimensions}

We summarize the generating solution for general rotating black holes which are electrically charged under the $U(1)$ gauge fields in heterotic string on tori, constructed in Ref. 40]. Such solutions are parameterized by the non-extremality parameter $m$, the angular momentum parameters $l_{i}\left(i=1, \ldots,\left[\frac{D-1}{2}\right]\right)$, and two electric charges $Q_{1}^{(1)}$ and $Q_{1}^{(2)}$, which correspond to the electric charges of a Kaluza-Klein and a two-form $U(1)$ gauge fields that are associated with the same compactification circle of the tori. Here, $m$ and $l_{i}$ are, respectively, related to the ADM mass and the angular momenta per unit ADM mass of the $D$-dimensional Kerr solution [46].

The generating solutions are constructed by imposing $S O(1,1)$ boost transformations in the $O(11-D, 27-D) T$-duality symmetry group of the $(D-1)$-dimensional action. The ADM mass $M$, the angular momenta $J_{i}$ and the electric charges of the generating solution in $D$ dimensions are given in terms of the parameters $\delta_{1}$ and $\delta_{2}$ of the $S O(1,1)$ boost transformations, and the parameters $m$ and $l_{i}$ by

$$
\begin{aligned}
M_{A D M} & =\frac{\Omega_{D-2} m}{8 \pi G_{D}}\left[(D-3)\left(\cosh ^{2} \delta_{1}+\cosh ^{2} \delta_{2}\right)-(D-4)\right], \\
J_{i} & =\frac{\Omega_{D-2}}{4 \pi G_{D}} m l_{i} \cosh \delta_{1} \cosh \delta_{2}, \\
Q_{1}^{(1)} & =\frac{\Omega_{D-2}}{8 \pi G_{D}}(D-3) m \cosh \delta_{1} \sinh \delta_{1}, \\
Q_{1}^{(2)} & =\frac{\Omega_{D-2}}{8 \pi G_{D}}(D-3) m \cosh \delta_{2} \sinh \delta_{2},
\end{aligned}
$$

where $\Omega_{D-2} \equiv \frac{2 \pi^{\frac{D-1}{2}}}{\Gamma\left(\frac{D-1}{2}\right)}$ is the area of a unit $(D-2)$-sphere and $G_{D}$ is the $D$-dimensional gravitational constant. Here, the $D$-dimensional gravitational constant $G_{D}$ is defined in terms of the ten-dimensional gravitational constant $G_{10}=8 \pi^{6} g_{s}^{2} \alpha^{\prime 4}$ as $G_{D}=G_{10} / V_{10-D}$, where $V_{10-D}$ is the volume of the $(10-D)$-dimensional internal space.

The Bekenstein-Hawking entropy $S_{B H}$ is determined by the following event horizon area $A_{D}$ through the relation $S_{B H}=\frac{A_{D}}{4 G_{D}}$ :

$$
A_{D}=2 m r_{H} \Omega_{D-2} \cosh \delta_{1} \cosh \delta_{2},
$$

where $r_{H}$ is the (outer) event horizon determined by the equation

$$
\left[\prod_{i=1}^{\left[\frac{D-1}{2}\right]}\left(r^{2}+l_{i}^{2}\right)-2 N\right]_{r=r_{H}}=0 .
$$

Here, $N$ is defined as $m r\left[m r^{2}\right]$ for even [odd] spacetime dimensions $D$.

The Hawking temperature $T_{H}=\frac{\kappa}{2 \pi}$ is determined by the following surface gravity $\kappa$ at the (outer) event horizon:

$$
\kappa=\left.\frac{1}{\cosh \delta_{1} \cosh \delta_{2}} \frac{\partial_{r}(\Pi-2 N)}{4 N}\right|_{r=r_{H}},
$$


where $\Pi \equiv \prod_{i=1}^{\left[\frac{D-1}{2}\right]}\left(r^{2}+l_{i}^{2}\right)$.

The following angular velocity $\Omega_{H i}\left(i=1, \ldots,\left[\frac{D-1}{2}\right]\right)$ of the event horizon is defined by the condition that the Killing vector $\xi \equiv \partial / \partial t+\Omega_{H i} \partial / \partial \phi_{i}$ is null on the event horizon, i.e. $\left.\xi^{\mu} \xi^{\nu} g_{\mu \nu}\right|_{r=r_{H}}=0$ :

$$
\Omega_{H i}=\frac{1}{\cosh \delta_{1} \cosh \delta_{2}} \frac{l_{i}}{r_{H}^{2}+l_{i}^{2}} .
$$

\section{B. Dyonic Rotating Black Hole in Heterotic String on a Six-Torus}

Rotating black hole solution in heterotic string on a six-torus constructed in Ref. [39] is parameterized by the non-extremality parameter $m$, the angular momentum $J$, a KaluzaKlein and a two-form electric charges $Q_{1}$ and $Q_{2}$ associated with the same compactification direction, and a Kaluza-Klein and a two-form magnetic charges $P_{1}$ and $P_{2}$ associated with the same compactification direction but different compactification direction from that of the electric charges.

In terms of the non-extremality parameter $m$, the rotational parameter $l$, and the boost parameters $\delta_{e 1}, \delta_{e 2}, \delta_{m 1}$ and $\delta_{m 2}$ of the $S O(1,1)$ boost transformations in the $O(8,24) U$ duality symmetry group of the heterotic string on a seven-torus, the ADM mass $M_{A D M}$, the angular momentum $J$, and electric and magnetic charges $Q_{1}, Q_{2}, P_{1}$ and $P_{2}$ are given by

$$
\begin{array}{rlrl}
M_{A D M} & =2 m\left(\cosh 2 \delta_{e 1}+\cosh 2 \delta_{e 2}+\cosh 2 \delta_{m 1}+\cosh 2 \delta_{m 2}\right), \\
J & =8 l m\left(\cosh \delta_{e 1} \cosh \delta_{e 2} \cosh \delta_{m 1} \cosh \delta_{m 2}-\sinh \delta_{e 1} \sinh \delta_{e 2} \sinh \delta_{m 1} \sinh \delta_{m 2}\right), \\
Q_{1} & =2 m \sinh 2 \delta_{e 1}, & Q_{2}=2 m \sinh 2 \delta_{e 2}, \\
P_{1} & =2 m \sinh 2 \delta_{m 1}, & P_{2}=2 m \sinh 2 \delta_{m 2} .
\end{array}
$$

The Bekenstein-Hawking entropy $S_{B H}=\frac{1}{4 G_{N}} A$ is determined by the surface area $A=$ $\left.\int d \theta d \phi \sqrt{g_{\theta \theta} g_{\phi \phi}}\right|_{r=r_{H}}$ of the (outer) event horizon at $r=r_{H}=m+\sqrt{m^{2}-l^{2}}$ as follows:

$$
\begin{aligned}
S & =16 \pi\left[m^{2}\left(\prod_{i=1}^{4} \cosh \delta_{i}+\prod_{i=1}^{4} \sinh \delta_{i}\right)+m \sqrt{m^{2}-l^{2}}\left(\prod_{i=1}^{4} \cosh \delta_{i}-\prod_{i=1}^{4} \sinh \delta_{i}\right)\right] \\
& =16 \pi\left[m^{2}\left(\prod_{i=1}^{4} \cosh \delta_{i}+\prod_{i=1}^{4} \sinh \delta_{i}\right)+\sqrt{m^{4}\left(\prod_{i=1}^{4} \cosh \delta_{i}-\prod_{i=1}^{4} \sinh \delta_{i}\right)^{2}-(J / 8)^{2}}\right.
\end{aligned}
$$

where $\delta_{1,2,3,4} \equiv \delta_{e 1, e 2, m 1, m 2}$.

\section{General Rotating Black Hole Solution in Heterotic String on a Five-Torus}

The generating solution for the general black hole solutions in heterotic string on a fivetorus constructed in Ref. [13] is parameterized by the non-extremality parameter $m$, two angular momenta $J_{1}$ and $J_{2}$, a Kaluza-Klein and the two-form electric charges $Q_{1}$ and $Q_{2}$ 
associated with the same compactification direction, and an electric charge $Q$ associated with the Hodge dual of the field strength of a two-form field in the NS-NS sector.

In terms of the non-extremality parameter $m$, the rotational parameters $l_{1}$ and $l_{2}$, and parameters $\delta_{1}, \delta_{2}$ and $\delta$ of the $S O(1,1)$ boost transformations in the $O(8,24) U$-duality group of heterotic string on a seven-torus, the ADM mass $M_{A D M}$, angular momenta $J_{1}$ and $J_{2}$, and electric charges $Q_{1}, Q_{2}$ and $Q$ are given by

$$
\begin{aligned}
M_{A D M} & =m\left(\cosh 2 \delta_{1}+\cosh 2 \delta_{2}+\cosh 2 \delta\right), \\
J_{1} & =4 m\left(l_{1} \cosh \delta_{1} \cosh \delta_{2} \cosh \delta-l_{2} \sinh \delta_{1} \sinh \delta_{2} \sinh \delta\right), \\
J_{2} & =4 m\left(l_{2} \cosh \delta_{1} \cosh \delta_{2} \cosh \delta-l_{1} \sinh \delta_{1} \sinh \delta_{2} \sinh \delta\right), \\
Q_{1} & =m \sinh 2 \delta_{1}, \quad Q_{2}=m \sinh 2 \delta_{2}, \quad Q=m \sinh 2 \delta
\end{aligned}
$$

The Bekenstein-Hawking entropy $S_{B H}=\frac{1}{4 G_{N}} A$ is determined by the surface area $A=$ $\left.\int d \theta d \phi_{1} d \phi_{2} \sqrt{g_{\theta \theta}\left(g_{\phi_{1} \phi_{1}} g_{\phi_{2} \phi_{2}}-g_{\phi_{1} \phi_{2}}^{2}\right)}\right|_{r=r_{H}}$ of the (outer) event horizon located at $r=r_{H}=$ $\left[m-\frac{1}{2} l_{1}^{2}-\frac{1}{2} l_{2}^{2}+\frac{1}{2} \sqrt{\left\{2 m-\left(l_{1}+l_{2}\right)^{2}\right\}\left\{2 m-\left(l_{1}-l_{2}\right)^{2}\right\}}\right]^{1 / 2}$ as follows:

$$
\begin{aligned}
S=4 \pi & {\left[m \sqrt{2 m-\left(l_{1}-l_{2}\right)^{2}}\left(\prod_{i=1}^{3} \cosh \delta_{i}+\prod_{i=1}^{3} \sinh \delta_{i}\right)\right.} \\
& \left.+m \sqrt{2 m-\left(l_{1}+l_{2}\right)^{2}}\left(\prod_{i=1}^{3} \cosh \delta_{i}-\prod_{i=1}^{3} \sinh \delta_{i}\right)\right] \\
=4 \pi & {\left[\sqrt{2 m^{3}\left(\prod_{i=1}^{3} \cosh \delta_{i}+\prod_{i=1}^{3} \sinh \delta_{i}\right)^{2}-\frac{1}{16}\left(J_{1}-J_{2}\right)^{2}}\right.} \\
& \left.+\sqrt{2 m^{3}\left(\prod_{i=1}^{3} \cosh \delta_{i}-\prod_{i=1}^{3} \sinh \delta_{i}\right)^{2}-\frac{1}{16}\left(J_{1}+J_{2}\right)^{2}}\right],
\end{aligned}
$$

where $\delta_{1,2,3} \equiv \delta_{1,2}, \delta$.

\section{STATISTICAL INTERPRETATION OF ROTATING BLACK HOLES IN HETEROTIC STRING ON TORI}

In this section, we elaborate on statistical interpretation of the Bekenstein-Hawking entropies of black hole solutions discussed in section $[\mathrm{IV}$. We find at least qualitative agreement between the Bekenstein-Hawking entropies and the statistical entropies based upon $D$-brane descriptions of Ref. [25,26] and the correspondence principle of Ref. [38]. We also speculate on the generalization of the Rindler space description of statistical entropy to the case of specific rotating black holes discussed in sections $\mathrm{IVB}$ and IV C.

\section{A. D-Brane Picture}

In this subsection, we attempt to give the statistical interpretation for the BekensteinHawking entropy of the non-extreme, rotating, five-dimensional and four-dimensional black 
holes discussed in section $\mathbb{Q}$ by applying $D$-brane description of black holes [26]. It turns out that even for non-extreme, rotating cases, the $D$-brane description reproduces the Bekenstein-Hawking entropy in the limit of large number of $D$-branes. This is expected from the perspective of the correspondence principle. In the following, we first discuss general formalism and then we consider the five-dimensional black hole and the four-dimensional black hole as special cases.

Generally, in the $D$-brane picture of black holes the statistical origin of the BekensteinHawking entropy is attributed to the oscillation degrees of freedom of open strings which stretch between $D$-branes. So, the statistical entropy of black holes is the logarithm of the asymptotic level density of open strings given in Eq.(33), which we shall write again here as

$$
S_{\text {stat }}=2 \pi \sqrt{\frac{c}{6}}\left[\sqrt{N_{L}}+\sqrt{N_{R}}\right],
$$

where $c=n_{b}+\frac{1}{2} n_{f}$ is the central charge determined by the bosonic $n_{b}$ and the fermionic $n_{f}$ degrees of freedoms for the configuration under consideration.

The oscillator levels $N_{L}$ and $N_{R}$ are determined by the level matching condition in terms of NS-NS electric charges and the non-extremality parameter. Since in the $D$-brane description of black holes $D$-branes do not play any dynamical role gravitationally, we assume that the ADM mass of the whole black hole configuration is the sum of the ADM mass of black hole that carries R-R charges and the mass of perturbative string states, as proposed in Refs. [27,29, 30. Of course, such an identification has to be made at the black hole and $D$-brane transition point, by following the correspondence principle of Ref. [38]. Then, equating the mass of the perturbative string states with the ADM mass of black holes which carry NS-NS electric charges, i.e. the Kaluza-Klein gauge field and the two-form gauge field electric charges, at the transition point, one obtains the oscillator levels $N_{L}$ and $N_{R}$ in terms of parameters of black hole solutions. The details are discussed in section $\nabla B$ and expressions for the five-dimensional and four-dimensional cases are given by:

$$
\begin{array}{lll}
N_{R} \approx \alpha^{\prime} m^{2} \cosh ^{2}\left(\delta_{2}-\delta_{1}\right), & N_{L} \approx \alpha^{\prime} m^{2} \cosh ^{2}\left(\delta_{2}+\delta_{1}\right), & \text { for 5-d case }, \\
N_{R} \approx 2 \alpha^{\prime} m^{2} \cosh ^{2}\left(\delta_{e 2}-\delta_{e 1}\right), & N_{L} \approx 2 \alpha^{\prime} m^{2} \cosh ^{2}\left(\delta_{e 2}+\delta_{e 1}\right), & \text { for 4-d case },
\end{array}
$$

where the boost parameters $\delta_{1}$ and $\delta_{2}$ are respectively associated with the Kaluza-Klein $U(1)$ gauge and the two-form $U(1)$ gauge electric charges, and $m$ is the non-extremality parameter.

The central charge $c$ is determined by the total number of bosonic and fermionic degrees of freedom within the configuration under consideration. It is in general expressed in terms of (the product of) the number of $D$-brane(s). We will give the expressions for $c$ in the following subsections when we consider specific configurations.

The effect of non-zero angular momenta on the statistical entropy of black holes can be explained in terms of conformal field theory technique as follows. The details are discussed for example in Refs. [47,48,26, 40]. So, we explain only the main points here. The spatial rotation group $S O(4)$, which is external to $D$-brane bound states, is isomorphic to the $S U(2)_{R} \times S U(2)_{L}$ group. This $S U(2)_{R} \times S U(2)_{L}$ group can be identified as the affine symmetry of $(4,4)$ superconformal algebra. The charges $\left(F_{R}, F_{L}\right)$ of the $U(1)_{R} \times U(1)_{L}$ subgroup in $S U(2)_{R} \times S U(2)_{L}$ group (which can be interpreted as the spins of string states) 
are related to the angular momenta $\left(J_{1}, J_{2}\right)$ of the rotational group $S O(4)$ in the following way

$$
J_{1}=\frac{1}{2}\left(F_{L}+F_{R}\right), \quad J_{2}=\frac{1}{2}\left(F_{R}-F_{L}\right)
$$

The $U(1)_{L, R}$ current $J_{L, R}$ can be bosonized as $J_{L, R}=\sqrt{\frac{c}{3}} \partial \phi$, and the conformal state $\Phi_{F_{L, R}}$ which carries $U(1)_{L, R}$ charge $F_{L, R}$ is obtained by applying an operator $\exp \left(\frac{i F_{L, R} \phi}{\sqrt{c / 3}}\right)$ to the state $\Phi_{0}$ without $U(1)_{L, R}$ charge. As a consequence, the conformal dimensions $h$ 's, i.e. the eigenvalues of the Virasoro generators $L_{0}$ and $\bar{L}_{0}$, of the two conformal fields $\Phi_{F_{L, R}}$ and $\Phi_{0}$ are related in the following way

$$
h_{\Phi_{F_{L}}}=h_{\Phi_{0}}+\frac{3 F_{L}^{2}}{2 c}, \quad h_{\Phi_{F_{R}}}=h_{\Phi_{0}}+\frac{3 F_{R}^{2}}{2 c} .
$$

This implies that the total number $N_{L_{0}}\left[N_{R_{0}}\right]$ of the left-moving [the right-moving] oscillations of spinless string states are reduced by the amount $\frac{3 F_{L}^{2}}{2 c}\left[\frac{3 F_{R}^{2}}{2 c}\right]$ in comparison with the total number $N_{L}\left[N_{R}\right]$ of the left-moving [the right-moving] oscillations of states with the specific spin $F_{L}\left[F_{R}\right]$ :

$$
\begin{aligned}
& N_{L} \rightarrow N_{L_{0}}=N_{L}-\frac{3 F_{L}^{2}}{2 c}, \\
& N_{R} \rightarrow N_{R_{0}}=N_{R}-\frac{3 F_{R}^{2}}{2 c} .
\end{aligned}
$$

Note, the level density $d_{0}$ for spinless states in a given level $\left(N_{L}, N_{R}\right)$ differs from the level density $d\left(N_{L}, N_{R}\right)=\exp \left[2 \pi \sqrt{\frac{c}{6}}\left(\sqrt{N_{L}}+\sqrt{N_{R}}\right)\right]$ of all the states in the level $\left(N_{L}, N_{R}\right)$ by a numerical factor, which can be neglected in the limit of large $\left(N_{L}, N_{R}\right)$ when one takes logarithm of the level density. Therefore, the statistical entropy of string states with specific spins $\left(F_{L}, F_{R}\right)$ is given by

$$
\begin{aligned}
& S_{\text {stat }} \simeq \ln d_{0}\left(N_{L_{0}}, N_{R_{0}}\right) \simeq 2 \pi \sqrt{\frac{c}{6}}\left(\sqrt{N_{L_{0}}}+\sqrt{N_{R_{0}}}\right) \\
& =2 \pi\left(\sqrt{\frac{c}{6} N_{L}-\frac{F_{L}^{2}}{4}}+\sqrt{\frac{c}{6} N_{R}-\frac{F_{R}^{2}}{4}}\right) \text {, }
\end{aligned}
$$

where $F_{R, L}=J_{1} \pm J_{2}$.

\section{Five-Dimensional Black Hole}

We consider the five-dimensional black hole discussed in section IVC. Black hole solutions in heterotic string on tori can be transformed into solutions in type-II string theories with R-R charges by applying the string-string duality between the heterotic string on $T^{4}$ and type-IIA string on $K 3, T$-duality between type-IIA string and type-IIB string (as necessary), and the $U$-duality of type-II string theory. Since such duality transformations leave 
the Einstein-frame metric intact, the Bekenstein-Hawking entropy has the same form after the duality transformations. The $D$-brane embedding of the five-dimensional black hole that we wish to consider in this section is the bound state of $Q_{5} D 5$-branes wrapped around a five-torus $T^{5}=T^{4} \times S^{1}$ and open strings wound around the circle $S^{1}$ with their internal momentum flowing along $S^{1}$. For this configuration, the central charge of open strings is given by $c=6 Q_{5}$, where $Q_{5}=m \sinh 2 \delta_{5}$. We assume that $Q_{5}$ is very large. Then, one has $Q_{5}=m \sinh 2 \delta_{5} \approx m \cosh ^{2} \delta_{5}$. Applying Eq.(57) with the central charge $c=6 Q_{5}$ and string oscillator levels given in Eq.(53), one has the following expression for statistical entropy of non-extreme, rotating, five-dimensional black hole:

$$
\begin{aligned}
S_{\text {stat }} \simeq & 2 \pi\left[\sqrt{\alpha^{\prime} m^{3} \cosh ^{2} \delta_{5}\left(\cosh \delta_{1} \cosh \delta_{2}+\sinh \delta_{1} \sinh \delta_{2}\right)^{2}-\frac{\left(J_{1}-J_{2}\right)^{2}}{4}}\right. \\
& \left.+\sqrt{\alpha^{\prime} m^{3} \cosh ^{2} \delta_{5}\left(\cosh \delta_{1} \cosh \delta_{2}-\sinh \delta_{1} \sinh \delta_{2}\right)^{2}-\frac{\left(J_{1}+J_{2}\right)^{2}}{4}}\right] .
\end{aligned}
$$

This agrees with the Bekenstein-Hawking entropy in Eq.(51) in the limit of large $Q$ (and therefore $\sinh \delta \simeq \cosh \delta$ ). The mismatch in factors in each term is due to difference in convention for defining $U(1)$ charges and angular momenta.

\section{Four-Dimensional Black Hole}

We discuss the statistical interpretation for the Bekenstein-Hawking entropy (49) of four-dimensional black hole solution in section $\mathbb{E V B}$. By applying duality transformations on this black hole solution, one can obtain a solution in type-II theory with R-R charges. In this section, we consider $D$-brane bound state corresponding to intersecting $Q_{6} D$ 6-branes and $Q_{2} D$ 2-branes which are respectively wrapped around a six-torus $T^{6}=T^{4} \times S_{1}^{\prime} \times S_{1}$ and the two-torus $T^{2}=S_{1}^{\prime} \times S_{1}$, and open strings wound around one of circles in the two-torus $T^{2}$, say $S_{1}$, with their momenta flowing along the same circle, i.e. $S_{1}$. The central charge of this $D$-brane bound state is given by $c=6 Q_{2} Q_{6}$, where in terms of boost parameters and the non-extremality parameter, the $D$-brane charges are given by $Q_{2}=$ $2 m \sinh 2 \delta_{D 2}$ and $Q_{6}=2 m \sinh 2 \delta_{D 6}$. For the four-dimensional black hole, the $U(1)_{L}$ charge $F_{L}$ is zero (therefore, $J:=J_{1}=J_{2}$ ), since only the right-moving supersymmetry survives (corresponding to the $(4,0)$ superconformal theory). We assume that $Q_{2}$ and $Q_{5}$ are very large, so that $Q_{5}=2 m \sinh 2 \delta_{D 5} \approx 2 m \cosh ^{2} \delta_{D 5}$ and $Q_{2}=2 m \sinh 2 \delta_{D 2} \approx 2 m \cosh ^{2} \delta_{D 2}$. Then, the general form of statistical entropy (57) reduces to the following form:

$$
\begin{aligned}
S_{\text {stat }} \simeq & 2 \pi\left[2 \sqrt{2} \sqrt{\alpha^{\prime}} m^{2} \cosh \delta_{D 2} \cosh \delta_{D 6}\left(\cosh \delta_{1} \cosh \delta_{2}+\sinh \delta_{1} \sinh \delta_{2}\right)\right. \\
& \left.+\sqrt{8 \alpha^{\prime} m^{4} \cosh ^{2} \delta_{D 2} \cosh ^{2} \delta_{D 6}\left(\cosh \delta_{1} \cosh \delta_{2}-\sinh \delta_{1} \sinh \delta_{2}\right)^{2}-J^{2}}\right] .
\end{aligned}
$$

This agrees with the Bekenstein-Hawking entropy (49) of the four-dimensional black hole solutions discussed in section IVB in the limit of large $P_{1}$ and $P_{2}$ (and therefore, $\sinh \delta_{m 1} \simeq$ $\cosh \delta_{m 1}$ and $\left.\sinh \delta_{m 2} \simeq \cosh \delta_{m 2}\right)$. 


\section{B. Correspondence Principle}

In this subsection, we generalize the corresponding principle of Ref. 38] to the case of rotating black holes. We consider the general class of electrically charged, rotating black holes in heterotic string on tori discussed in section IVA, and we shall find that statistical entropy from correspondence principle is in qualitative agreement with the Bekenstein-Hawking entropy of such solutions.

According to $D$-brane or fundamental string description of black holes, black holes are regarded as the strong string coupling limit of the perturbative string states or the bound states of $D$-branes. Namely, since the gravitational constant is proportional to the square of string coupling constant, when string coupling is very large the strong gravitational field causes gravitational collapse, leading to the formation of black hole. On the other hand, for a small value of string coupling, spacetime approaches flat spacetime and the theory is described by perturbative strings or $D$-branes. Therefore, at the particular value of string coupling there exists the transition point between black holes and perturbative $D$-brane or string descriptions. It is claimed in Ref. 38 that this occurs when the curvature at the event horizon of a black hole is of the order of string scale $l_{s} \approx \sqrt{\alpha^{\prime}}$ or equivalently when the size of the event horizon is of the order of string scale, i.e. $r_{H} \sim \sqrt{\alpha^{\prime}}$. At the transition point, the mass of perturbative string states can be equated with the ADM mass of black holes, making it possible to apply the level matching condition.

First, we relate the macroscopic quantities that characterize black holes to the microscopic quantities of perturbative strings by applying the level matching condition. According to the correspondence principle, this is possible when the size of the event horizon is of the order of the string length scale, i.e. $r_{H} \sim \sqrt{\alpha^{\prime}}$. Since we are considering black hole solutions with the Kaluza-Klein and the two-form electric charges associated with the same compactification direction, the corresponding Virasoro condition for perturbative string states is the one for string compactified on a circle of radius $R$ :

$$
M_{s t r}^{2}=p_{R}^{2}+\frac{4}{\alpha^{\prime}} N_{R}=p_{L}^{2}+\frac{4}{\alpha^{\prime}} N_{L}
$$

where $M_{s t r}$ is the mass of string states, $p_{R, L}=\frac{n_{w} R}{\alpha^{\prime}} \pm \frac{n_{p}}{R}$ is the right (left) moving momentum in the direction of circle, and $N_{R, L}$ is the right (left) moving oscillator level. Here, $n_{w}$ and $n_{p}$ are respectively the string winding number and the momentum quantum number along the direction of the circle. The Kaluza-Klein electric charge and the two-form electric charge of black holes in string theory are respectively identified with the momentum and the winding modes of strings in the compact direction. Therefore, the right and left moving momenta of strings in the compact direction are given in terms of parameters of black hole solutions discussed in section $\mathrm{DA}$ by

$$
p_{R, L}=\frac{\Omega_{D-2}}{8 \pi G_{D}}(D-3) m\left(\cosh \delta_{2} \sinh \delta_{2} \pm \cosh \delta_{1} \sinh \delta_{1}\right) .
$$

When the size of the event horizon is of the length of string scale (i.e. $r_{H} \sim \sqrt{\alpha^{\prime}}$ ), one can further identify the ADM mass of the black hole with the mass of string states, i.e. $M_{s t r} \simeq \frac{\Omega_{D-2} m}{8 \pi G_{D}}\left[(D-3)\left(\cosh ^{2} \delta_{1}+\cosh ^{2} \delta_{2}\right)-(D-4)\right]$. Then, from the level matching 
condition (60) with (61) substituted one obtains the following expressions for the right and left moving oscillation levels in terms of parameters of black hole solution

$$
\begin{aligned}
& N_{R} \approx \frac{\alpha^{\prime}}{4}\left(\frac{\Omega_{D-2}}{8 \pi G_{D}}\right)^{2}(D-3)^{2} m^{2} \cosh ^{2}\left(\delta_{2}-\delta_{1}\right) \\
& N_{L} \approx \frac{\alpha^{\prime}}{4}\left(\frac{\Omega_{D-2}}{8 \pi G_{D}}\right)^{2}(D-3)^{2} m^{2} \cosh ^{2}\left(\delta_{2}+\delta_{1}\right)
\end{aligned}
$$

in the limit of large electric charges $Q_{1}$ and $Q_{2}$.

The statistical entropy of the black hole is given by the logarithm of the degeneracy of string states. From the expression for oscillation levels in Eq. 62 one obtains the following form of statistical entropy:

$$
\begin{aligned}
S_{\text {stat }} & =2 \pi \sqrt{\frac{c}{6}}\left(\sqrt{N_{L}}+\sqrt{N_{R}}\right) \\
& \simeq \frac{(D-3) \Omega_{D-2} \sqrt{\alpha^{\prime}} m}{4 G_{D}} \sqrt{\frac{c}{6}} \cosh \delta_{1} \cosh \delta_{2},
\end{aligned}
$$

in the limit of large electric charges. On the other hand, at the transition point, the Bekenstein-Hawking entropy (44) takes the following form:

$$
S_{B H}=\frac{A_{D}}{4 G_{D}} \simeq \frac{m \sqrt{\alpha^{\prime}} \Omega_{D-2}}{2 G_{D}} \cosh \delta_{1} \cosh \delta_{2} .
$$

Here, $m$ is a function of angular momentum parameters $l_{i}$ and $\alpha^{\prime}$, and is determined by the

equation $\prod_{i=1}^{\left[\frac{D-1}{2}\right]}\left(\alpha^{\prime}+l_{i}^{2}\right)-2 N=0$. Therefore, the Bekenstein-Hawking entropy (64) and the statistical entropy (63) agree up to a numerical factor of order one.

\section{Statistical Entropy and Rindler Geometry}

As for the statistical interpretation of rotating black hole entropy based upon Rindler space description, which is extensively discussed in the previous sections, the author does not have a complete understanding yet. But if such description is a right interpretation of black hole entropy, we believe that the entropy of rotating black holes is nothing but the statistical entropy of a gas of strings which rotate with rotating black holes that carry (the remaining) non-perturbative charges. In the following subsection, we shall discuss necessary ingredients for understanding the Rindler spacetime description for the general class of four-dimensional and five-dimensional black holes discussed in section IV.

\section{Five-Dimensional Black Hole}

The five-dimensional black hole solution constructed in Ref. [13 carries two electric charges $Q_{1}$ and $Q_{2}$ of a Kaluza-Klein $U(1)$ gauge field and a two-form $U(1)$ gauge field in the NS-NS sector, and one electric charge $Q$ associated with the Hodge-dual of the field strength of the two-form field in the NS-NS sector. We interpret the statistical origin of 
entropy of this black hole solution as being due to the microscopic degrees of freedom of gas of perturbative strings with momentum number $Q_{1}$ and the winding number $Q_{2}$ which oscillate in the background of black hole with electric charge $Q$.

Five-dimensional, rotating black hole with the electric charge $Q$ has the surface gravity at the event horizon $r=r_{H}$ given by

$$
\begin{aligned}
\kappa & =\frac{1}{\cosh \delta} \frac{2 r_{H}^{2}+l_{1}^{2}+l_{2}^{2}-2 m}{2 m r_{H}} \\
& =\frac{1}{m \cosh \delta} \frac{\sqrt{\left\{2 m-\left(l_{1}+l_{2}\right)^{2}\right\}\left\{2 m-\left(l_{1}-l_{2}\right)^{2}\right\}}}{\sqrt{2 m-\left(l_{1}+l_{2}\right)^{2}}+\sqrt{2 m-\left(l_{1}-l_{2}\right)^{2}}} .
\end{aligned}
$$

The Rindler observer will detect thermal radiation of a gas of strings with temperature $T=\frac{\kappa}{2 \pi}$. The total energy of strings in the comoving frame with metric given by Eq.(đ) is related to the Rindler energy of strings in the frame with metric (21) by the factor of the above surface gravity $\kappa$ at the event horizon.

Note, in the extreme limit the surface gravity $\kappa$ vanishes, leading to infinite rescaling of oscillation levels or infinite statistical entropy. This is not surprising since in general in the extreme limit observers in the comoving frame with angular velocity of event horizon have to move faster than the speed of light, i.e. $g_{t t}^{\prime} \geq 0$ for $r \geq r_{H}$, and as a consequence the statistical observerbles are not well-defined. So, the statistical description in the comoving frame which rotates with angular velocity of the event horizon cannot be applied to the extreme rotating black holes.

By applying the level matching condition, one can see that the right moving and the left moving oscillation levels $N_{R}$ and $N_{L}$ (of free strings in the Minkowski background) are given by

$$
N_{R} \approx \alpha^{\prime} m^{2} \cosh ^{2}\left(\delta_{2}-\delta_{1}\right), \quad N_{L} \approx \alpha^{\prime} m^{2} \cosh ^{2}\left(\delta_{2}+\delta_{1}\right)
$$

in the limit of large $Q_{1}$ and $Q_{2}$. Naively just by applying the prescription of Ref. [27,29, 30, i.e. the statistical entropy is given by the logarithm of level density with string oscillation levels $N_{L}$ and $N_{R}$ rescaled by the surface gravity $\kappa$, one does not reproduce the BekensteinHawking entropy. We speculate that the left-moving and the right-moving oscillator levels $N_{L}$ and $N_{R}$ should be rescaled by different factors since the left-moving and the rightmoving sectors of string gas form separate non-interacting thermal systems with different temperatures $T_{L}$ and $T_{R}$. The following rescalings of the oscillator levels $N_{L}$ and $N_{R}$ would yield the correct expressions for statistical entropy:

$$
\begin{aligned}
& N_{L} \rightarrow N_{L}^{\prime}=N_{L}\left[2 m-\left(l_{1}-l_{2}\right)^{2}\right] \cosh ^{2} \delta, \\
& N_{R} \rightarrow N_{R}^{\prime}=N_{R}\left[2 m-\left(l_{1}+l_{2}\right)^{2}\right] \cosh ^{2} \delta,
\end{aligned}
$$

for the five-dimensional black holes with large $Q=m \sinh 2 \delta$. These rescaling factors cannot be understood from the temperatures $T_{L}$ and $T_{R}$ of the left-movers and the right-movers alone. So, we believe that there are some subtle points in statistical mechanics of string gas in the Rindler frame that we do not have a complete understanding of. 


\section{Four-Dimensional Black Hole}

The four-dimensional black hole solution constructed in Ref. [39] is charged under two electric charges $Q_{1}$ and $Q_{2}$ of a Kaluza-Klein $U(1)$ gauge field and a two-form $U(1)$ gauge field in the NS-NS sector, and under two magnetic charges $P_{1}$ and $P_{2}$ of a Kaluza-Klein $U(1)$ gauge field and a two-form $U(1)$ gauge field in the NS-NS sector. Just as in the fivedimensional case in section $\mathrm{VC}$, we attribute the statistical entropy of this black hole as being due to a gas of perturbative strings with momentum number $Q_{1}$ and the winding number $Q_{2}$ which oscillate in the background of black hole that carries magnetic charges $P_{1}$ and $P_{2}$.

The surface gravity at the event horizon $r=r_{H}$ of the four-dimensional, rotating black hole with magnetic charges $P_{1}$ and $P_{2}$ is given by

$$
\kappa=\frac{1}{\cosh \delta_{m 1} \cosh \delta_{m 2}} \frac{r_{H}-m}{2 m r_{H}}=\frac{1}{2 m \cosh \delta_{m 1} \cosh \delta_{m 2}} \frac{\sqrt{m^{2}-l^{2}}}{m+\sqrt{m^{2}-l^{2}}} .
$$

As expected, in the extreme limit $(m=l)$ the analysis of this section cannot be applied, since $\kappa=0$.

By applying the level matching condition, one obtains the following expression for the oscillator levels for free strings in the Minkowski background:

$$
N_{R} \approx 2 \alpha^{\prime} m^{2} \cosh ^{2}\left(\delta_{e 2}-\delta_{e 1}\right), \quad N_{L} \approx 2 \alpha^{\prime} m^{2} \cosh ^{2}\left(\delta_{e 2}+\delta_{e 1}\right),
$$

in the limit of large $Q_{1}$ and $Q_{2}$. We speculate that in the throat region of comoving frame the string oscillation levels $N_{L}$ and $N_{R}$ are rescaled in the following way:

$$
\begin{aligned}
& N_{L} \rightarrow N_{L}^{\prime}=4 m^{2} \cosh ^{2} \delta_{m 1} \cosh ^{2} \delta_{m 2} N_{L}, \\
& N_{R} \rightarrow N_{R}^{\prime}=4\left(m^{2}-l^{2}\right) \cosh ^{2} \delta_{m 1} \cosh ^{2} \delta_{m 2} N_{R} .
\end{aligned}
$$

for the four-dimensional black hole with large $P_{1}=2 m \sinh 2 \delta_{m 1}$ and $P_{2}=2 m \sinh 2 \delta_{m 2}$.

\section{ACKNOWLEDGMENTS}

The work is supported by U.S. DOE Grant No. DOE DE-FG02-90ER40542. 


\section{REFERENCES}

[1] J.D. Bekenstein, Phys. Rev. D7 (1973) 2333.

[2] J.D. Bekenstein J.D. 1974, Phys. Rev. D9 (1974) 3292.

[3] S.W. Hawking, Phys. Rev. Lett. 26 (1971) 1344.

[4] S.W. Hawking, Phys. Rev. D13 (1976) 191.

[5] J.M. Bardeen, B. Carter and S.W. Hawking, Comm. Math. Phys. 31 (1973) 161.

[6] G. 't Hooft G. 1985, Nucl. Phys. B256 (1985) 727.

[7] L. Susskind, Preprint RU-93-44, hep-th/9309145.

[8] L. Susskind and J. Uglum, Phys. Rev. D50 (1994) 2700.

[9] M.J. Duff and J. Rahmfeld, Phys. Lett. B345 (1995) 441.

[10] A. Dabholkar, J.P. Gauntlett, J.A. Harvey and D. Waldram, Nucl. Phys. B474 (1996) 85.

[11] A. Sen, Mod. Phys. Lett. A10 (1995) 2081.

[12] M. Cvetič M. and D. Youm, Phys. Rev. D53 (1995) 584.

[13] M. Cvetic and D. Youm, Nucl. Phys. B476 (1996) 118.

[14] M. Cvetic and A.A. Tseytlin, Phys. Rev. D53 5619, Erratum-ibid. D 55 (1997) 3907.

[15] A.A. Tseytlin, Mod. Phys. Lett. A11 (1996) 689.

[16] A.A. Tseytlin, Nucl. Phys. B477 (1996) 431.

[17] J. Polchinski, Phys. Rev. Lett. 75 (1995) 4724.

[18] J. Dai, R.G. Leigh and J. Polchinski, Mod. Phys. Lett. A4 (1989) 2073.

[19] A. Sen, Phys. Rev. D54 (1996) 2964.

[20] A. Sen, Phys. Rev. D53 (1996) 2874.

[21] C. Vafa, Nucl. Phys. B463 (1996) 415.

[22] C. Vafa, Nucl. Phys. B463 (1996) 435.

[23] M. Bershadsky, V. Sadov and C. Vafa, Nucl. Phys. B463 (1996) 398.

[24] M. Bershadsky, V. Sadov and C. Vafa 1996, Nucl. Phys. B463 (1996) 420.

[25] A. Strominger and C. Vafa, Phys. Lett. B379 (1996) 99.

[26] C.G. Callan and J.M. Maldacena, Nucl. Phys. B472 (1996) 591.

[27] E. Halyo, A. Rajaraman and L. Susskind, Phys. Lett. B392 (1997) 319.

[28] E. Halyo, B. Kol, A. Rajaraman and L. Susskind, Preprint SU-ITP-96-40, hepth/9609075.

[29] E. Halyo, Preprint SU-ITP-42, hep-th/9610068.

[30] E. Halyo, Preprint SU-ITP-96-51, hep-th/9611175.

[31] J.M. Maldacena and A. Strominger, Phys. Rev. Lett. 77 (1996) 428.

[32] A. Dabholkar, Preprint TIFR-TH-97-02, hep-th/9702050.

[33] G.T. Horowitz and A. Strominger, Phys. Rev. Lett. 77 (1996) 2368.

[34] J.C. Breckenridge, D.A. Lowe, R.C. Myers, A.W. Peet, A. Strominger and C. Vafa, Phys. Lett. B381 (1996) 423.

[35] G.T. Horowitz, D.A. Lowe and J.M. Maldacena, Phys. Rev. Lett. 77 (1996) 430.

[36] G.T. Horowitz, J.M. Maldacena and A. Strominger, Phys. Lett. B383 (1995) 151.

[37] J.M. Maldacena, Preprint RU-96-102, hep-th/9611125.

[38] G.T. Horowitz and J. Polchinski, Phys.Rev. D55 (1997) 6189.

[39] M. Cvetic and D. Youm, Phys. Rev. D54 (1996) 2612.

[40] M. Cvetic and D. Youm, Nucl. Phys. B477 (1996) 449. 
[41] K. Huang and S. Weinberg, Phys. Rev. Lett. 25 (1970) 895.

[42] R. Hagedorn, Suppl. Nuovo Cim. 3 (1965) 147.

[43] R. Hagedorn, Astron. Astropys. 5 (1970) 184.

[44] J.M. Maldacena and L. Susskind, Nucl. Phys. B475 (1996) 679.

[45] S.P. de Alwis and K. Sato, Phys. Rev. D55 (1997) 6181.

[46] R.C. Myers and M.J. Perry, Annals Phys. 172 (1986) 304.

[47] J.C. Breckenridge, R.C. Myers, A.W. Peet and C. Vafa, Phys. Lett. B391 (1997) 93.

[48] C. Vafa and E. Witten, Nucl. Phys. B431 (1994) 3. 